Artículo de Investigación

\title{
Las políticas públicas de desarrollo empresarial e innovación desde la perspectiva de la nueva gestión pública y la gobernanza: caso CREAME *
}

\author{
Public policies of business development and \\ innovation from the perspective of new public \\ management and governance: case CREAM E
}

Geovanny Perdomo Charry** José Enrique Arias Pérez***

Fecha de recepción: 6 de mayo de 2013

Fecha de aprobación: 29 de mayo de 2013

* Este artículo de investigación es producto de la tesis doctoral "Análisis del cambio organizacional e institucional. Estudio comparado de dos incubadoras de empresas: "Centro Integral de Servicios Empresariales (CREAME) en Medellín, Colombia (1996-2010) y Barcelona Activa, en Barcelona, España (1986-2010)".

** Doctor en Administración, Universidad EAFIT; magíster en Gerencia del Talento Humano, Universidad de Manizales; especialista en Derecho Comercial y Financiero, Universidad Católica de Colombia; administrador de empresas, Universidad Cooperativa de Colombia;profesor, Departamento de Ciencias Administrativas, Universidad de Antioquia. Medellín- Colombia. Correo electrónico:gperdomo@eafit.edu.co

*** Magíster en Gestión de Ciencia, Tecnología, Universidad de Antioquia;especialista en Gerencia de Sistemas y Tecnología, Universidad Pontificia Bolivariana; administrador de empresas, Fundación Universitaria Luis Amigó; profesor, Departamento de Ciencias Administrativas, Universidad de Antioquia. Medellín-Colombia. Correo electrónico: joenriquearias@posgradoseconomicas.com 
Las políticas públicas de desarrollo empresarial e innovación desde la perspectiva

Geovanny Perdomo Charry - J osé Enrique A rias Pérez

\section{Resumen}

El objetivo del artículo es analizar las políticas públicas de desarrollo empresarial e innovación, desde la perspectiva de la nueva gestión pública, la gobernanza y de una incubadora de empresas de gran incidencia en su formulación, CREAME. En la metodología se utilizó el estudio de caso, combinando el análisis documental con la entrevista semiestructurada. En los resultados se evidencia que las políticas han surgido predominantemente en el contexto regional, por la convergencia de diversos actores, entre ellos los no gubernamentales, que juegan un papel importante en la formulación, ejecución y evaluación; lo cual coincide con los rasgos del modelo de gestión pública modus vivendi o negociado de acción.

Palabras clave: emprendimiento, gestión de innovación, gestión pública, gobernanza, incubadora de empresas, sistema regional de innovación.

Clasificación JEL: L 26, 0 32, L53, G 30, G34, 039

\section{Abstract}

The aim of this paper is to analyze public policies of business development and innovation, from the perspective of the new public management, governance and a business incubator that has a large impact on its formulation, CREAM E. In the methodology, we used the case study, combining documentary analysis with semi-structured interview. The results evidenced that policies have emerged predominantly in the regional context, by the convergence of different actors, including non governmental, that play an important role in the formulation, implementation and evaluation, which is consistent with the features of the public management model modus vivendi and/ or the negotiated of action.

Keywords: business incubator, regional system of innovation, entrepreneurship, public management, governance, innovation management.

JEL Classification: L26, 0 32, L53, G 30, G34, 039 


\section{INTRODUCCIÓN}

En Colombia, las incubadoras de empresas se crean a partir de la Ley de Fomento de la Investigación Científica y el Desarrollo Tecnológico, Ley 29 de 1990. Y el sistema nacional de ciencia, tecnología e innovación (SNCTI) empieza a fortalecerse aún más, a partir de la modificación de la Ley 29 de 1990 por la Ley 1286 de 2009, pues se transforma a Colciencias en Departamento Administrativo y se robustece el sistema en Colombia, que está integrado por tres tipos legales de conformación organizacional: público, privado y mixto, que han evolucionado a nivel nacional y regional.

En el marco del proceso de fortalecimiento y desarrollo del sistema nacional y regional de ciencia, tecnología e innovación (SNCTI y SRI) en Colombia por factores endógenos y exógenos, las incubadoras han tenido particular atención en la Ley
1286 de 2009 de fomento de la ciencia, la tecnología y la innovación, cuando se expresa de forma taxativa en su artículo 17, cómo puede institucionalizarse la noción de ciencia, tecnología e innovación en el sistema, con la decidida participación, colaboración, articulación e interrelación de los diversos actores del sistema en función de un objetivo común: mejorar los niveles de productividad y competitividad del sector empresarial colombiano. En esta Ley se señala, como uno de sus objetivos principales, dentro del artículo 17 , numeral 2:

Fomentar y consolidar, con visión de largo plazo los centros y grupos de investigación particulares y de las Instituciones de Educación Superior (...); las incubadoras de empresas de base tecnológica y el desarrollo del talento humano; las academias y sociedades científicas, tecnológicas y 
de innovación; y las diferentes redes, iniciativas de organizaciones e individuos tendientes al fortalecimiento del sistema (p. 2).

Lo anterior ha hecho que el SNCTI y el SRI en Colombia se desarrollen a partir de factores endógenos y exógenos. Esta evolución se debe a la complejidad de la interrelación y colaboración que existe entre los distintos actores y sistemas, ya sea por su carácter, objeto y función dentro del mismo sistema. Por tanto, es importante señalar que a partir de 2002, el Servicio Nacional de Aprendizaje (SENA), al percibir dificultades de articulación, coordinación e integración de esfuerzos entre las incubadoras, decidió incorporar dentro de su política el programa nacional de creación e incubación de empresas con el fin de iniciar un proceso de apoyo y fortalecimiento de las incubadoras en el territorio nacional, que ayudara a mejorar las relaciones e interacciones entre ellas y el sistema.

Por ello, se puede señalar que el origen y la proliferación de las incubadoras en Colombia se remonta a 1994, con el primer modelo de incubación de empresas que surgió con la Corporación Innovar en Bogotá y fue continuado por otras ocho incubadoras, incluida CREAME, que es el nombre de la organización objeto de estudio en este artículo. Es durante la década del noventa que los planes, las políticas y los programas de desarrollo empresarial, diseñados e implantados por el gobierno colombiano, se orientan a la creación de empresas y al fortalecimiento empresarial desde la política pública institucional, mediante el control nacional de las incubadoras, al ligar la evaluación del desempeño a las políticas de financiamiento a través del programa nacional de creación e incubación de empresas. No ocurre así con el factor económico, en que el financiamiento de las incubadoras se ha dirigido hacia la autofinanciación de las mismas y se percibe muy poco apoyo económico en el escenario nacional y regional para ejercer su labor principal: promover, fomentar y acompañar la creación de empresas y el fortalecimiento empresarial, pues dirigen con más fuerza su labor hacia la gestión y ejecución de proyectos que le permitan la autosostenibilidad, descuidando en gran parte su función u objeto central.

En el fondo, el avance del desarrollo empresarial y la innovación resulta de la convergencia de diversos factores, entre ellos, la interrelación de actores, instituciones, niveles de gobierno y políticas públicas que han determinado durante las últimas dos décadas el desarrollo del SNCTI y de las incubadoras, como lo señalan los últimos artículos científicos elaborados en Colombia sobre esta temática (Garrido, 2003; Malaver \& Vargas, 2005; Malaver \& Vargas, 2006; Monroy, 2006; Montenegro, 2007; Orozco, 2006; Robledo \& Echavarría, 2006).

Uno de los factores poco estudiados, corresponde a las políticas públicas de desarrollo empresarial e innovación (Monroy, 2006; Malaver \& Vargas, 2005; 
Orozco, 2006; Robledo y Echavarría, 2006); por ende, este artículo se ocupa de analizar su evolución desde la teoría de la nueva gestión pública y la gobernanza aplicado a un actor de gran incidencia en la formulación de las mismas en el ámbito regional, a saber, CREAME -incubadora de empresas-.

En ese orden de ideas, el presente artículoaborda, en líneas generales y en primer lugar, la definición de incubadora de empresa; en segundo lugar realiza una aproximación teórica a las políticas públicas, la nueva gestión pública y la gobernanza; después señala la metodología utilizada, esto es, el estudio de caso que combina el análisis documental con el análisis de información cualitativa de fuentes primarias; posteriormente se presentan los resultados y las conclusiones.

\section{APROXIMACIÓN A LA DEFI- NICIÓN DE INCUBADORA DE EMPRESA}

En la Tabla 1 se observa el tránsito que ha sufrido la noción de incubadora de empresas, que hasta los años ochenta era concebida desde un entorno organizacional y después de la década de los noventa hasta hoy, como un entorno institucional. Así, su concepción inicial pasa de estudiarse como ámbito organizacional que nutre la creación de nuevos negocios, a verse más como aquella institución capaz de efectuar procesos dinámicos, dirigidos por un entorno social, económico y político que facilita la aceleración, el descubrimiento, la validación y la aplicación de nuevas ideas y conceptos, con el fin de desarrollar y comercializar nuevos productos, tecnologías y negocios.

Por esta razón, es primordial enunciar algunos elementos clave de la noción de incubadoras siguiendo el orden de referencia de los actores, y así contrastar las diferentes perspectivas teóricas citadas para dar un concepto de la misma desde otro punto de vista, que intente complementar las definiciones actuales.

Los términos más asociados a cada concepto son: apoyo al inicio o creación de empresas, servicios de asistencia y recursos compartidos, supervivencia, red de apoyo o sistema de incubación, aceleración, facilidades e instalaciones para sus incubados, vulnerabilidad y programa de incubadoras de empresas. Teniendo en cuenta los diferentes elementos y términos asociados con la concepción de incubadora de empresas, para esta investigación se propone definir una incubadora de empresas como un medio formal dentro de un entorno circundante, 'una comunidad dentro de la comunidad', que genera fuentes críticas de oportunidades y restricciones a través de su red de apoyo, donde la disponibilidad, la accesibilidad y el aprovisionamiento de servicios y recursos compartidos facilita el inicio, el desarrollo, la aceleración y la supervivencia de negocios emprendedores, destinados a comercializar nuevas tecnologías, productos y servicios. 
Las políticas públicas de desarrollo empresarial e innovación desde la perspectiva Geovanny Perdomo Charry - J osé Enrique A rias Pérez

Tabla1. Definiciones del concepto de incubadora de empresas

\begin{tabular}{|c|c|}
\hline Autores & Definición \\
\hline $\begin{array}{l}\text { Allen } \\
\text { (1985) }\end{array}$ & $\begin{array}{l}\text { Son instalaciones que prometen y proporcionan a las empresas, en la } \\
\text { fase inicial de crecimiento, un espacio alquilado con servicios compar- } \\
\text { tidos de oficina, de negocios y de consultoría. }\end{array}$ \\
\hline $\begin{array}{l}\text { NBIA } \\
\text { (1985) }\end{array}$ & $\begin{array}{l}\text { Es una herramienta de desarrollo económico, diseñado para acelerar el } \\
\text { crecimiento y el éxito de las empresas mediante una serie de recursos de } \\
\text { apoyo empresarial y de servicios, puestos en marcha por la administra- } \\
\text { ción y que son ofrecidos por la incubadora de empresas y la red de } \\
\text { contactos. }\end{array}$ \\
\hline $\begin{array}{c}\text { Smillor \& Gill } \\
\text { (1986) }\end{array}$ & $\begin{array}{l}\text { Un programa de incubación tecnológica es un sistema de innovación } \\
\text { diseñado para asistir al emprendedor en el desarrollo de su nueva em- } \\
\text { presa de base tecnológica, en la creación de nuevas y novatas empre- } \\
\text { sas. Este tiene por objeto establecer un enlace efectivo entre personas, } \\
\text { tecnología, capital y conocimiento, aprovechando el talento empresa- } \\
\text { rial con el fin de acelerar el desarrollo de nuevas compañías, comercia- } \\
\text { lizando velozmente la tecnología. }\end{array}$ \\
\hline $\begin{array}{c}\text { Campbell } \\
\text { (1989) }\end{array}$ & $\begin{array}{l}\text { El rol formal de las incubadoras de empresas es ser "agentes de cam- } \\
\text { bio" en el desarrollo de la economía, capaces de hacer frente a fallas del } \\
\text { mercado entre ellas, los altos costos de la información, los bajos nive- } \\
\text { les de servicioy la escasez de fuentes de capital. }\end{array}$ \\
\hline $\begin{array}{l}\text { Allen \& } \\
\text { McCluskey } \\
\text { (1990) }\end{array}$ & $\begin{array}{l}\text { Es una instalación que proporciona el espacio económico, de servicios } \\
\text { compartidos de oficina y asistencia en el desarrollo del negocio en un } \\
\text { ambiente conducido para la creación y supervivencia de nuevas em- } \\
\text { presas en una corta etapa de crecimiento. }\end{array}$ \\
\hline $\begin{array}{l}\text { NBIA } \\
\text { (1996) }\end{array}$ & $\begin{array}{l}\text { Son programas de asistencia para negocios globales, que tienen por } \\
\text { objeto ayudar y soportar procesos de inicio o etapas tempranas para } \\
\text { empresas incipientes, con el objetivo de promover su crecimiento de } \\
\text { una manera viable, autosostenible y como un negocio financieramente } \\
\text { independiente (Adkins, 1996, citado por Eshun, 2004, p. 62). }\end{array}$ \\
\hline $\begin{array}{l}\text { Sherman \& } \\
\text { Chappell } \\
\text { (1998) }\end{array}$ & $\begin{array}{l}\text { Los programas de incubadoras de empresas son definidos como aque- } \\
\text { llos miembros actuales de NBIA y que habían estado en funcionamien- } \\
\text { to durante un período de al menos cinco años. Adicionalmente, estos } \\
\text { han de gestionar sobre el terreno la orientación proporcionada de su } \\
\text { gestión, asistencia técnica, alquiler de espacio, servicio de consultoría } \\
\text { a clientes, servicios empresariales compartidos y equipos básicos que } \\
\text { tienen declarada una política de graduación. }\end{array}$ \\
\hline $\begin{array}{l}\text { Hansen, Nohria } \\
\text { \& Nerger } \\
\text { (2000) }\end{array}$ & $\begin{array}{l}\text { Hacen hincapié en la posición facilitadora de las incubadoras de nego- } \\
\text { cios en aprovisionar a las empresas para que encuentren la manera de } \\
\text { surgir y recoger frutos en el futuro. }\end{array}$ \\
\hline
\end{tabular}




\begin{tabular}{|c|l|}
\hline Autores & \multicolumn{1}{c|}{ Definición } \\
\hline $\begin{array}{c}\text { Eshun } \\
(2004)\end{array}$ & $\begin{array}{l}\text { Es el entorno diseñado para estimular el crecimiento y desarrollo de } \\
\text { empresas en estado infantil, mediante la implementación de oportuni- } \\
\text { dades de adquisición y explotación de recursos con el fin de facilitar o } \\
\text { acelerar el desarrollo y la comercialización de nuevas ideas y conceptos } \\
\text { de negocios. }\end{array}$ \\
\hline $\begin{array}{c}\text { Hackett \& Dilts } \\
\text { (2004ay 2004b) }\end{array}$ & $\begin{array}{l}\text { Un centro de incubación de empresas, es un espacio u oficina compar- } \\
\text { tida que pretende ofrecer a sus incubados un sistema de intervención } \\
\text { estratégica y de valor añadido para la asistencia y seguimiento de ne- } \\
\text { gocios (...) con el fin de facilitar el desarrollo exitoso de la empresa y al } \\
\text { mismo tiempo vincular el costo de su fracaso potencial (...) Es una red } \\
\text { de individuos y organizaciones. }\end{array}$ \\
\hline $\begin{array}{l}\text { Abduh, Souza, } \\
\text { Quazi \& Burley }\end{array}$ & $\begin{array}{l}\text { Una incubadora de empresas o el programa o sistema de incubación de } \\
\text { empresas se perciben como una estrategia de desarrollo de las empre- } \\
\text { sas destinadas a acelerar el proceso de formación, desarrollo y supervi- } \\
\text { vencia de nuevas empresas en la comunidad. }\end{array}$ \\
\hline Tsai, Hseih, & $\begin{array}{l}\text { Las incubadoras de empresas proporcionan recursos y conocimientos } \\
\text { a sus inquilinos, que a menudo son jóvenes y carecen de recursos } \\
\text { críticos para la comercialización de sus productos o servicios. Se des- } \\
\text { criben como procesos de valor agregado, actividades, acuerdos, pro- } \\
\text { gramas diseñados y servicios que ofrecen las incubadoras de empre- } \\
\text { sas para facilitar y acelerar el desarrollo de los incubados hasta el punto } \\
\text { en que pueden salir de la incubadora y competir de manera eficiente e } \\
\text { independiente en un mercado dinámico. }\end{array}$ \\
\hline $\begin{array}{l}\text { Es un medio formal para estimular el crecimiento y el desarrollo de } \\
\text { nuevas empresas en etapa temprana mediante la mejora de sus oportu- } \\
\text { nidades, para la adquisición de los recursos destinados a facilitar el } \\
\text { desarrollo y la comercialización de nuevos productos, tecnologías y } \\
\text { modelos de negocio. Define,también, las incubadoras de empresas como } \\
\text { un proceso social y de gestión destinado a apoyar el desarrollo y la } \\
\text { comercialización de nuevos productos, tecnologías y modelos de ne- } \\
\text { gocio. }\end{array}$ \\
\hline Eshun
\end{tabular}

Fuente: elaborado por los autores, con base en Verma (2004).

POLÍTICAS PÚBLICAS, NUEVA GESTIÓN PÚBLICA Y GOBERNANZA

Dado que en esta investigación se entienden las políticas públicas como configuraciones de los actores (Muller, 2006), es necesario atender una diversidad de objetos de estudio -organizaciones públicas y gubernamentales y organizaciones no gubernamentales, entre otros-, que están implicados en la formulación, ejecución y evaluación de políticas públicas en materia de desarrollo empresarial e innovación. 
Por tanto, antes de describir los conceptos, niveles y categorías de análisis, presentaremos de manera sintética las actividades decisorias y los puntos de configuración de la política pública, por un lado, y por otro, los modelos y fases de la nueva gestión pública y cómo se asumen las tres nociones expuestas (políticas públicas, nueva gestión pública y gobernanza), que para este estudio se abordan desde la concepción de las políticas públicas de Meny y Thoening (1992) y Muller (2006), la nueva gestión pública de Monnier (1992) y la gobernanza en el análisis institucional desde Peters (2004) y Ramírez, Vargas y De la Rosa (2011).

Tabla 2. Actividades decisorias y puntos para la configuración de la política pública

\begin{tabular}{|c|c|c|}
\hline Aspecto & Descripción & Clasificación \\
\hline \multirow[t]{2}{*}{$\begin{array}{l}\text { Actividades } \\
\text { decisorias }\end{array}$} & \multirow{2}{*}{$\begin{array}{l}\text { Un enfoque corriente consiste } \\
\text { en inventariar y nombrar las ac- } \\
\text { tividades o fases elementales ne- } \\
\text { cesarias para la constitución del } \\
\text { llamado proceso de toma de de- } \\
\text { cisiones públicas (Lasswell, } \\
\text { 1979; Brewer, De Léon, 1983), } \\
\text { citados por Meny y Thoening } \\
\text { (1992, p. 136). Así, el decisor } \\
\text { maneja a la vez, un contenido, } \\
\text { problema -preferencias del } \\
\text { decisor-y un proceso, situación } \\
\text { o contexto -obligaciones-. }\end{array}$} & $\begin{array}{l}\text { La fase de formulación designa el trabajo mediante el } \\
\text { cual una apuesta se inscribe en la agenda gubernamen- } \\
\text { tal o un problema se transforma en alternativas para la } \\
\text { acción, en soluciones (Meny \& Thoening, 1992, p. } \\
\text { 136). }\end{array}$ \\
\hline & & $\begin{array}{l}\text { La fase de legitimación parece más breve y más iden- } \\
\text { tificable. En el sentido estricto del término, coincide } \\
\text { con el responsable formal, el individuo o el grupo al } \\
\text { que institucionalmente le corresponde el derecho y el } \\
\text { deber de resolver (Meny \& Thoening, 1992, p. 137). }\end{array}$ \\
\hline $\begin{array}{c}\text { Puntos } \\
\text { para la } \\
\text { configuración }\end{array}$ & $\begin{array}{l}\text { Las políticas públicas "no son } \\
\text { solamente un proceso de deci- } \\
\text { sión, sino el lugar donde una } \\
\text { sociedad dada construye su re- } \\
\text { lación con el mundo. Una polí- } \\
\text { tica pública, es entonces, tam- } \\
\text { bién la construcción de una ima- } \\
\text { gen de la realidad sobre la cual } \\
\text { se quiere intervenir" (Muller, } \\
\text { 2006, p. 95). }\end{array}$ & $\begin{array}{l}\text { El problema de la racionalidad de los actores, señala } \\
\text { la incertidumbre y la complejidad de los procesos de } \\
\text { decisión, basados principalmente en la noción de ra- } \\
\text { cionalidad limitada con autores clásicos como Simon, } \\
\text { Lindblomo Cohen, March y Olsen, citados por Muller } \\
\text { (2006, p. 67). } \\
\text { El papel de la administración pública como medio } \\
\text { decisional, está configurado por cuatro círculos de de- } \\
\text { cisión: administradores centrales, administradores sec- } \\
\text { toriales, socios externos del Estado y órganos políti- } \\
\text { cos, por ejemplo el Congreso (Muller, 2006, p. 68). } \\
\text { Las redes de actores se constituyen en redes de políti- } \\
\text { cas públicas. Allí, se trata de identificar los actores } \\
\text { susceptibles de actuar en la interfaz entre las diferen- } \\
\text { tes redes, en la medida que serán ellas quienes ejerce- } \\
\text { rán la función estratégica de integración de las diferen- } \\
\text { tes dimensiones de la decisión (Muller, 2006, p. 69). }\end{array}$ \\
\hline
\end{tabular}

Fuente: elaborado por los autores, con base en Meny y Thoening (1992) y Muller (2006). 
Para el análisis de las políticas y programas en materia de desarrollo empresarial e innovación en esta investigación, se propone, en primer lugar, describir el proceso de formulación y legitimación de la toma de decisiones del sector público y privado, así como los puntos que tuvieron en cuenta los actores sociales para configurar dichas políticas y programas, toda vez que "el proceso de decisión pública implica institucionalizar cambios en la forma de gobernar y tomar decisiones para impulsar el desarrollo local" (Rosas, 2011, p. 2); dicha decisión pública afecta de forma directa e indirecta a los incubados, las incubadoras, la red y la comunidad.

De esta manera, las políticas públicas son vistas como acciones y toma de decisiones de los servidores públicos. En palabras de Meny y Thoening:

La persona que decide no está sola, ni es omnipotente; lejos de ello, en su sustancia, el poder se reparte entre numerosos jugadores, cuyo peso varía según las apuestas que les atañen. Hay un juego en la sociedad política, y el decisor formal no es libre. Más aún, los hilos de la decisión no los manipulan los mismos jugadores. El poder es difuso y el juego, pluralista, La decisión pública es un compromiso entre múltiples actores interdependientes (1992, p. 130).

Así mismo, los factoresque provocan la reforma política son las condiciones socio-históricas (consciencia de la problemática), contar con una personalidad reformadora (confianza en el gobierno) y tener un estilo de decisión (valores y principios, por ejemplo igualdad en el acceso al emprendimiento y su financiación). Esto significa, que: "la acción política interviene de manera intermitente para señalar el objetivo general y, después, para arbitrar las medidas tomadas al detalle" (Meny \& Thoening, 1992, p. 134). Precisamente, en el contexto de estos dos aspectos, en la Tabla 3 se exponen los modelos y fases de configuración de la nueva gestión pública y un programa de acción.

De la Tabla 3 se adopta para esta investigación el modelo de gestión pública de modus vivendi o negociado de acción, dado que: "el origen de una acción se da como respuesta a un acontecimiento repentino, a una crisis o al incremento progresivo del interés ciudadano por un tema" (Monnier, 1992, p. 120), que para este caso en particular, es el desarrollo empresarial y la innovación.

Dicho modelo asigna un lugar privilegiado a la naturaleza de las interacciones en el seno de una misma organización y entre organizaciones. El enfoque sistémico, concretamente el de Crozier y Friedberg (1990), hace hincapié en las relaciones de interdependencia, pese a no haber valorado siempre sus consecuencias: la dependencia genera conflictos y "obliga a las partes a negociar" (Monnier, 1992, p. 106). 
Las políticas públicas de desarrollo empresarial e innovación desde la perspectiva Geovanny Perdomo Charry - J osé Enrique A rias Pérez

Tabla 3. Modelos y fases de configuración de la nueva gestión pública

\begin{tabular}{|c|c|c|}
\hline Aspecto & Descripción & Clasificación \\
\hline \multirow[t]{3}{*}{$\begin{array}{c}\text { Modelos } \\
\text { de gestión } \\
\text { pública }\end{array}$} & \multirow{3}{*}{$\begin{array}{l}\text { Descripción } \\
\text { La pretensión de proponer un } \\
\text { procedimiento formal para con- } \\
\text { cebir y ejecutar acciones políti- } \\
\text { cas carece de sentido, pues nin- } \\
\text { gún actor controla totalmente } \\
\text { los procesos en marcha } \\
\text { (Monnier, 1992, p. 89). Inclu- } \\
\text { so, se reconoce que los modelos } \\
\text { de administración y gestión pú- } \\
\text { blica han transitado y al mismo } \\
\text { tiempo habitado en tres mode- } \\
\text { los diferentes, pero comple- } \\
\text { mentarios. }\end{array}$} & $\begin{array}{l}\text { Clasificación } \\
\text { El modelo jurídico proscribe toda interacción entre la } \\
\text { sociedad civil y el Estado. La actuación estatal se ajus- } \\
\text { ta exclusivamente a derecho y las instancias elegidas } \\
\text { deciden libremente qué marco jurídico garantizará el } \\
\text { interés público (Monnier, 1992, p. 97). }\end{array}$ \\
\hline & & $\begin{array}{l}\text { El modelo técnico-económico viene a ser tan normati- } \\
\text { vo como el anterior, porque establece reglas de funcio- } \\
\text { namiento que, sin igualar el formalismo de las reglas } \\
\text { jurídicas, pueden resultar tan uniformadoras. A los } \\
\text { valores tradicionales de seguridad, continuidad, regu- } \\
\text { laridad, se superponen ahora los de eficacia y rentabi- } \\
\text { lidad (Monnier, 1992, p. 101). }\end{array}$ \\
\hline & & $\begin{array}{l}\text { El modelo modus vivendi o negociado de acción, per- } \\
\text { mite reflejar mejor, a nuestro juicio, la realidad de la } \\
\text { acción de los poderes públicos, ya que se apoya en el } \\
\text { principio de que la decisión es fruto de una cadena de } \\
\text { negociaciones intra e interorganizacionales, de que la } \\
\text { acción deriva de las interacciones entre los actores so- } \\
\text { ciales y, por último, de que cualquier política expresa } \\
\text { un compromiso temporal, que se materializa en un } \\
\text { programa de acción (Monnier, 1992, p. 110). }\end{array}$ \\
\hline \multirow[t]{3}{*}{$\begin{array}{c}\text { Fases de un } \\
\text { programa } \\
\text { de acción }\end{array}$} & \multirow{3}{*}{$\begin{array}{l}\text { Toda acción política se propo- } \\
\text { ne la resolución de un problema } \\
\text { social cuya formulación se ex- } \\
\text { presa en términos más o menos } \\
\text { claros y se evalúa de forma } \\
\text { pluralista donde se asocian los } \\
\text { actores con el desarrollo de la } \\
\text { evaluación, a fin de incrementar } \\
\text { su credibilidad y, consecuente- } \\
\text { mente su utilidad social } \\
\text { (Monnier, 1992, p. 123-130). }\end{array}$} & $\begin{array}{l}\text { La fase uno es el conocimiento previo sobre el campo } \\
\text { de acción considerado. Esto dependerá, en efecto, de } \\
\text { la capacidad de los actores para concebir una teoría de } \\
\text { acción pertinente -Causal Theory-, es decir, un proto- } \\
\text { tipo inicial de la acción inicial ola formulación de un(os) } \\
\text { programa(s) de acción (Monnier, 1992, p. 124). }\end{array}$ \\
\hline & & $\begin{array}{l}\text { La dos expresa que la existencia de medios de acción } \\
\text { apropiados depende de la capacidad de los actores } \\
\text { para superar los obstáculos técnicos, financieros, jurí- } \\
\text { dicos, económicos, que se logra a partir de la puesta a } \\
\text { punto del programa y el test de difusión o comunica- } \\
\text { ción posterior (Monnier, 1992, p. 124). }\end{array}$ \\
\hline & & $\begin{array}{l}\text { La tercera recuerda que dependiendo de la homogenei- } \\
\text { dad y de la accesibilidad de los destinatarios resultará } \\
\text { más o menos fácil concebir el programa adecuado para } \\
\text { convertirlo en rutina y evaluarlo. Los tres tipos de } \\
\text { evaluación son: la previa o de diagnóstico (evaluación } \\
\text { a priori, prospectiva o ex ante), seguimiento o evalua- } \\
\text { ción concomitante y finalmente un balance o evalua- } \\
\text { ción a posteriori (Monnier, 1992, p. 125). }\end{array}$ \\
\hline
\end{tabular}

Fuente: elaborado por los autores, con base en Monnier (1992). 
Por tanto, si se asume que una organización y un programa son ante todo espacios para negociar más o menos institucionalizados, entonces deben concurrir muchas mediaciones para que se alcance un compromiso(Monnier, 1992, p. 119), lo cual implica que la reformulación, la traducción y las sucesivas negociaciones contribuyen a definir progresivamente los programas de acción por fases (formulación, ejecución y evaluación), en respuesta a la demanda social o de mercado.

Por ende, la nueva gestión pública se define: "con la idea de que el mecanismo de mercado, siempre que sea posible, es el mejor componente para distribuir los beneficios vía eficiencia técnica, descuidando en parte la noción política" (Arellano \& Cabrero, 2005, p. 602). La noción política entonces, se puede "explicar a través de la complementariedad social que retoma la gestión pública desde el desempeño organizacional a la capacidad institucional" (Ramírez, Vargas \& De la Rosa, 2011, p. 35).

De otra parte la gobernanza se entiende como una serie de mecanismos que proveen servicios públicos a ciertos espacios sociales (Peters, 2004, p. 69). Dichos espacios se definen, no obstante, en otros términos, ya no desde un punto de vista secuencial, sino en virtud de la naturaleza de las interacciones entre los actores sociales (contexto). Estos: "campos de interacción son, respectivamente, el contexto de la legitimación (legitimidad jurídica, política, científica-técnica, operativa y demográfica), el contexto de la acción programada (autonomía relativa) y, por último, el de la reacción o contexto de la evaluación espontánea, implícita o formalizada" (Monnier, 1992, p. 117).

El análisis de la gobernanza "se ha dedicado en mayor parte a examinar el papel que desempeñan los actores no gubernamentales en la producción de las políticas públicas" (Peters, 2004, p. 69). No es posible pensar la gobernanza sin gobierno, es decir, ver los actores públicos y privados involucrados en la elaboración de políticas, pero orientados, dirigidos y evaluados por el gobierno mismo. En las dos transformaciones del sistema político y administrativo se encuentra la institucionalización de la burocracia y los acuerdos de la administración pública.

La posibilidad de hacer una reforma de la administración pública al estilo de la gobernanza, dependerá mucho de que existan organizaciones fuertes en la sociedad civil, así como normas institucionalizadas que legitimen la participación directa de los grupos de interés y otros organismos de análisis en la elaboración de políticas públicas (Peters, 2004, p. 95). Con base en lo anterior, se puede señalar que existen algunos fundamentos teóricos adoptados en las políticas públicas, la nueva gestión pública y la gobernanza, que permiten proponer una relación entre dos niveles de análisis (red y comunidad) y las categorías de análisis de estas tres nociones (ver Tabla 4). 
Las políticas públicas de desarrollo empresarial e innovación desde la perspectiva Geovanny Perdomo Charry - J osé Enrique A rias Pérez

Tabla 4. Nivel y categorías de análisis de las políticas y programas de desarrollo empresarial e innovación

\begin{tabular}{|c|c|c|}
\hline Perspectiva & Nivel & Categorías de análisis \\
\hline \multirow{5}{*}{$\begin{array}{l}\text { Políticas } \\
\text { públicas, nueva } \\
\text { gestión pública } \\
\text { y gobernanza }\end{array}$} & \multirow[t]{2}{*}{$\begin{array}{l}\text { Meso: } \\
\text { Red }\end{array}$} & $\begin{array}{l}\text { Desempeño organizacional: enlos estudios } \\
\text { organizacionales "se identifican tres enfoques de des- } \\
\text { empeño: capacidad de producir valor a los clientes, los } \\
\text { asociados a los logros individuales y colectivos y final- } \\
\text { mente, la perspectiva interna y externa. Los dos prime- } \\
\text { ros se ubican en el ámbito más racionalista de la discipli- } \\
\text { na administrativa, mientras que el tercero trata de dar } \\
\text { una visión más dinámica y amplia; se ubica más en la } \\
\text { perspectiva de los Estudios Organizacionales" (Ramírez, } \\
\text { Vargas \& De la Rosa, 2011, p. 27). }\end{array}$ \\
\hline & & $\begin{array}{l}\text { Administración y planeación estratégica: se puede "afir- } \\
\text { mar que mientras la Planeación Estratégica se desarrolla } \\
\text { a luz de la Teoría de la Organización, la Administración } \\
\text { Estratégica surge a la luz de los Estudios } \\
\text { Organizacionales. Sin el desarrollo de la Teoría de la Or- } \\
\text { ganización y los Estudios Organizacionales, la Planeación } \\
\text { Estratégica y la Administración Estratégica, difícilmente } \\
\text { hubieran evolucionado hacia el Pensamiento Estratégi- } \\
\text { co tal y como se conocen hoy en día" (Ramírez, Vargas \& } \\
\text { De la Rosa, 2011, p. 25). }\end{array}$ \\
\hline & \multirow[t]{3}{*}{$\begin{array}{c}\text { Macro: } \\
\text { comunidad }\end{array}$} & $\begin{array}{l}\text { Gobernabilidad: el gobierno no debe soslayarse como } \\
\text { uno de los actores principales en esta función de con- } \\
\text { ducción social. En este sentido, el gobierno es una con- } \\
\text { dición necesaria pero no suficiente en el proceso de di- } \\
\text { rección de la sociedad, por más que se trate de un go- } \\
\text { bierno capaz, efectivo y eficiente (Aguilar, 2006, citado } \\
\text { por Ramírez, Vargas \& De la Rosa, 2011, p. 39). }\end{array}$ \\
\hline & & $\begin{array}{l}\text { Gobernanza: significa un "proceso de dirección de la } \\
\text { sociedad que no es equivalente a la sola acción directiva } \\
\text { del gobierno sino del conjunto social" (Ramírez, Vargas } \\
\text { \& De la Rosa, 2011, p. 39). }\end{array}$ \\
\hline & & $\begin{array}{l}\text { Capacidad institucional: es vistacomo "la condensación } \\
\text { compleja de recursos, mecanismos y dispositivos tanto } \\
\text { de orden técnico y organizacional como de naturaleza } \\
\text { política, de los que un gobierno dispone para la conduc- } \\
\text { ción de la acción social o colectiva orientada al desarro- } \\
\text { llo y bienestar de una comunidad o territorio, histórica y } \\
\text { socialmente determinados" (Ramírez, Vargas \& De la } \\
\text { Rosa, 2011, p. 40). }\end{array}$ \\
\hline
\end{tabular}

Fuente: elaborado por los autores, con base en Ramírez, Vargas y De la Rosa (2011). 


\section{METODOLOGÍA}

Antes de iniciar el trabajo de campo para la recolección de la información, y como lo señala Yin en su modelo: "en la etapa de definición y diseño es necesario el diseño del protocolo de recolección de datos" (2003, p. 50), que se refiere a la elaboración de un documento que incluye los pasos e instrumentos por utilizar para la recolección de datos en cada una de las actividades. Son ellos:definición y diseño, preparación, recolección y tratamiento, así como análisis y conclusiones.

\section{Definición y diseño del caso: CREAME}

La primera etapa, denominada aplicación teórica, se lleva por medio de una validación tanto teórica, como de expertos de la incubadora y el entorno, con el fin de construir un consenso generalizado sobre los factores internos y externos (incluyen políticas y programas) más desarrollados por las instituciones durante las dos últimas décadas, para luego incorporarlos en los instrumentos de revisión jurídica y legal, revisión documental y entrevistas. Este ejercicio ayudó a superar las limitaciones de cultura y ambiente de esta investigación.

En el interior de este proceso, antes de la aplicación de los instrumentos, se efectuaron dos actividades concretas; la primera estuvo relacionada con la revisión de las seis revistas más representativas del campo del emprendimiento que estudian los factores que inciden o condicionan la actividad emprendedora y la creación de empresas. El segundo aspecto se desarrolla con base en los elementos encontrados en la revisión de la literatura, para posteriormente elaborar y validar los instrumentos con la ayuda de grupo de académicos y expertos.

\section{Preparación, recolección y trata-} miento de los datos en CREAME

Dentro de esta etapa se considera importante hacer, en primer término, una breve descripción de CREAME, que vincula su historia, trayectoria $\mathrm{y}$ transformación como un centro integral de servicios empresariales y más concretamente su labor como incubadora de empresas. Posteriormente se analizan las características, la oferta y la demanda de productos o servicios, así como los factores internos y externos que inciden en su proceso de cambio y transformación. Así, entonces, el protocolo estructura de forma operativa las fuentes primarias y secundarias, el método de recolección, las categorías estudiadas, como el tiempo y el procedimiento de análisis (ver Tabla 5). 
Las políticas públicas de desarrollo empresarial e innovación desde la perspectiva

Geovanny Perdomo Charry - J osé Enrique A rias Pérez

Tabla 5. Diseño de recolección de datos del caso empírico

\begin{tabular}{|c|c|c|}
\hline Aspectos & Descripción & Duración \\
\hline $\begin{array}{l}\text { Fuentes de } \\
\text { información } \\
\text { y población } \\
\text { objetivo. }\end{array}$ & $\begin{array}{l}\text { - Primarias: grupo de personas vinculadas a la organi- } \\
\text { zación en la dinámica y transformación de la incuba- } \\
\text { dora de empresas: directivos (2), empleados (9) e in- } \\
\text { cubados (9). } \\
\text { - Secundarias: folletos informativos, página web, ac- } \\
\text { tas, memorias de la organización, informes de gestión, } \\
\text { portafolios de servicios, documentos de planeación y } \\
\text { estadísticas de la incubadora, entre otras. }\end{array}$ & $1 \mathrm{mes}$ \\
\hline $\begin{array}{l}\text { Método de } \\
\text { recolección de } \\
\text { información } \\
\text { y categorías } \\
\text { estudiadas. }\end{array}$ & $\begin{array}{l}\text { - Revisión jurídica y legal: estádirigida a recolectar toda } \\
\text { la información del entorno acerca de normas y leyes } \\
\text { en ciencia, tecnología e innovación y desarrollo em- } \\
\text { presarial; políticas y programas en esta misma mate- } \\
\text { ria, y factores contextuales. Duración: un mes en el } \\
\text { entorno investigado. } \\
\text { - Revisión documental: estáorientada a recolectar toda } \\
\text { la información de la organización sobre: antecedentes } \\
\text { e hitos históricos, factores internos y factores exter- } \\
\text { nos. Duración: un mes en la organización. } \\
\text { - Entrevistassemiestructuradas a los tres grupos de } \\
\text { personas: 1) Características, funciones y roles de la } \\
\text { organización en el proceso de cambio. 2) Factores in- } \\
\text { ternos. 3) Factores externos -incluso políticas y pro- } \\
\text { gramas-. }\end{array}$ & 3 meses \\
\hline $\begin{array}{l}\text { Procedimiento } \\
\text { de análisis. }\end{array}$ & $\begin{array}{l}\text { - Análisis descriptivo del caso según el procedimiento } \\
\text { de "análisis simple de casos" con el apoyo de un soft- } \\
\text { ware de análisis de información cualitativa. }\end{array}$ & $1 \mathrm{mes}$ \\
\hline
\end{tabular}

Fuente: elaborado por los autores.

Para llevar a cabo de manera apropiada el tratamiento de los datos, se recurre al apoyo técnico de las entrevistas, que consta del formato estructurado con las preguntas, para realizar su seguimiento estricto, y una grabadora digital, para hacer el registro de cada respuesta. Después, el investigador principal transcribe las respuestas en archivos independientes, y luego de cada entrevista se efectúa una revisión para confirmar la información y corregir posibles problemas inherentes a la transcripción.

En este sentido, las preguntas son categorizadas en tres secciones que indagan por antecedentes, características y funciones de la organización, y políticas y programas, las cuales, según los atributos de cada miembro o usuario de la incubadora, se contestan en un número determinado, como se puede observar en la Tabla 6. 
Tabla 6. Número de preguntas por cada sección según el atributo del actor

\begin{tabular}{|l|c|c|c|}
\hline Secciones & Directivos & Empleados & Incubados \\
\hline Sección 1: Antecedentes, & & & \\
características y funciones & 3 & 2 & 5 \\
Sección 2: Políticas & 3 & 6 & 8 \\
Sección 3: Programas & 3 & 3 & 3 \\
\hline
\end{tabular}

Fuente: elaborado por los autores.

De esta manera, dentro de las diferentes entrevistas, las secciones corresponden a diversas categorías de análisis como etapas del proceso de cambio y transformación de la incubadora. En ellas se indaga a algunos miembros (directivos y empleados) y usuarios (incubados) sobre algunos elementos relacionados con su origen, desarrollo, cambio y transformación sufrido por la incubadora y la incidencia que han tenido los factores y las políticas y programas en este cambio.

Los datos recolectados por medio de veinte entrevistas (dos directivos, nueve empleados y nueve incubados), se analizan a través de un software de procesamiento de información cualitativa, en donde, una vez realizadas, se transcriben en un procesador de palabras similar a Word. Después estas son trasladadas al programa, para ser reformadas en estructura de párrafo y convertidas a un formato de texto numerado que permite obtener archivos con líneas numeradas a las cuales son aplicados los códigos (categorías de análisis) mediante la lectura completa de todo el archivo.
En este proceso de codificación se establece una relación de los textos con las categorías, de manera tal que lleve al programa a la generación de salidas a través de un proceso de búsquedas, categoría por categoría, en las que el investigador, a medida que revisa el comportamiento de las mismas, establece su análisis de acuerdo con la necesidad que proponga la investigación (similitudes, diferencias, convergencias, consensos y disensos, entre otros).

\section{Análisis y reporte del caso CREAME}

La etapa 3, "análisis y reporte del caso de estudio", se desarrolla por una parte, con el software, y, por otra, con el procedimiento de análisis simple de casos de Yin (2003, p.68), que da lugar al paso final deanálisis de las políticas públicas de desarrollo empresarial e innovación en relación con la incubadora de empresas CREAME.

\section{RESULTADOS}

CREAMEsurge como Incubadora de Empresas de Base Tecnológica de Antioquia (IEBTA) el 27 de marzo de 
1996, fecha en que obtiene personería jurídica quedando registrada en el acta No. 001 de constitución de la corporación. No obstante, es el 22 de julio de 1996, cuando realmente se cierra su legalización en la escritura de constitución, acto formal que tuvo lugar en la Gobernación de Antioquia. Así mismo, el 19 de noviembre del mismo año, se le otorga la personería jurídica por parte de la Gobernación, mediante escritura pública levantada en la Notaría 20 de Medellín ${ }^{1}$ y registrada en la Cámara de Comercio de la ciudad.

Dichas organizaciones se asociaron en una figura mixta dando origen a IEBTA, a través de cuatro principales fuentes de recursos: un aporte económico único de $50 \mathrm{smmlv}^{2}$ en efectivo; un soporte en redes de evaluación, investigación y referencia; recursos en especie, resaltando la adjudicación que hizo Empresas Públicas de Medellín en diciembre de 1996, cuando definió formalmente aportar un edificio ubicado en la calle 11 con carrera 43B en el barrio El Poblado, y finalmente, el establecimiento de lazos de cooperación internacional con una serie de países que han avanzado en este tipo de alianzas institucionales.

En la entrevista a los miembros del cuadro directivo de CREAME, se destaca significativamente la cooperación privada que ha tenido la corporación, y se subraya la formidable apertura de apoyo de las empresas antioqueñas, acudiendo a las solicitudes de verificación y consultoría tecnológica en temas inherentes al trabajo particular en formulación de proyectos de la incubadora. Igualmente, se observa que la incubadora contó con el acompañamiento especial de la Promotora de Proyectos de Antioquia S.A. (PRO ANTIOQUIA) y la colaboración y el acompañamiento del Centro Tecnológico de Antioquia (CTA), que administró y tuteló durante 1996 los recursos de la misma.

En cuanto a las actividades generales de funcionamiento y puesta en marcha de la incubadora, se dio inicio a procesos de identificación y complementación interinstitucional, incorporando las $\mathrm{ONG}$ y algunas corporaciones como: a) Actuar Famiempresas, b) Innotech: convenio para la asignación de formuladores de los

\footnotetext{
$1 \quad$ Con la escritura de constitución No. 3650 del 30 de agosto de 1996, se crea la Corporación de Incubadora de Empresas de Base Tecnológica de Antioquia, ante la notaría 20 del círculo notarial de Medellín, cuyos participantes fueron: Universidad de Antioquia, Universidad EAFIT, Universidad Pontificia Javeriana, Universidad de Medellín, Politécnico Colombiano Jaime Isaza Cadavid, Universidad Nacional de Colombia Seccional Medellín, Corporación Universitaria Lasallista de Medellín, Universidad Católica de Oriente, Instituto Tecnológico Metropolitano, Escuela de Ingeniería de Antioquia; y compañías como: Confecciones Leonisa S.A., Compañía de Cementos Argos S.A., Sociedad Festo Limitada, Cámara de Comercio de Medellín, Federación Nacional de Cafeteros de Colombia, Asociación de Industriales -ANDI-, Fundación Corona, Corporación Financiera Nacional Suramericana S.A. -CORFINSURA-, y Fundación para el Desarrollo de Antioquia -PROANTIOQUIA-. Igualmente, la incubadora recibió de Colciencias, el SENA y el Ministerio de Desarrollo apoyo económico para promover su labor en el municipio de Medellín, mediante acuerdos de cooperación-cofinanciación de proyectos de los incubados y de programas especiales de la incubadora-.

2 Salarios mínimos mensuales legales vigentes.
} 
proyectos y realización del Censo Biotecnológico de Antioquia; c) Corporación Minuto de Dios; d) Asociación de Instructores y Técnicos Pensionados del SENA: convenio de provisión de cooperación experta nacional; e) CIB y f) Instituto de Medicina Tropical.

Otras de las organizaciones e instituciones que han ayudado al fortalecimiento de la incubadora, son el CONPES de Occidente, con la inserción en la región occidental del país; el Comité Consultivo de Países de la Cuenca del Pacífico; la Corporación Innovar, incubadora de empresas de base tecnológica de Bogotá; COMFAMA, con el manejo consensualizado y capacitación calificada de recursos humanos; COMFENALCO; y los foros permanentes de centros de investigación y desarrollo e incubadoras de empresas, coordinados por Colciencias (CREAME, 1998).

Incluso dentro de las actividades específicas de la Corporación, se pusieron en marcha algunos programas especiales como la elaboración de la propuesta de estatutos, las reuniones del comité promotor, la elaboración de proyectos con destino a Colciencias y el Instituto de Financiamiento Industrial -IFI-, el desarrollo de dos foros con los representantes de $\mathrm{I}+\mathrm{D}$ de las empresas antioqueñas, la participación en programas especiales, el diseño del plan de trabajo con las universidades, las redes de capacitación, información, soporte y evaluación primaria, las temáticas relacionadas con la propiedad industrial, el desarrollo del seminario regional con el IFI, la cátedra de emprendimiento empresarial, el trabajo con los comités de profesores y estudiantes, los comités de biotecnología, y el trabajo en equipo con los comités de microelectrónica.

Otra actividad primordial estuvo relacionada con los procesos de formación en este campo del saber, que emergieron a partir del diseño e implementación de la red de formulación integrada por estudiantes de último semestre y recién egresados, quienes fueron acompañados por tutores y por la red general de la incubadora, dando lugar a la creación de un sistema de extensión y representación de esta en las instituciones educativas y las empresas.

Como resultado del diseño y desarrollo del modelo de incubación (preincubaciónincubación-postincubación) en los dos primeros años (1996-1998), se inicia la etapa de preincubados e incubados con 31 proyectos, logrando durante el periodo 1999-2005, sensibilizar a la comunidad acerca de la necesidad de emprender y establecer alianzas con grandes compañías como Oracle, Asemarcas IBM, Corfinsura, SBI, SENA, Gobierno Alemán (CREAME, 1998). A la par del desarrollo y la consolidación de la IEBTA, se inicia la transferencia del modelo de Incubación a las ciudades de Pereira, Barranquilla, Manizales y Cartagena, así como algunos desarrollos con multinacionales de tecnología y 
fondos de capital de riesgo (CREAME, 1999).

Durante los siguientes diez años de desarrollo (de 1996 a 2005), la IEBTA le entregó a la región una corporación líder en emprendimiento, autosostenible y reconocida por el cumplimiento de su función, con logros evidentes. A lo largo de su trayectoria, la incubadora ha contribuido a la creación y el fortalecimiento de 937 empresas en las áreas de base tecnológica, agroindustria y valor agregado (CREAME, 2006).

En los siguientes tres años (2006-2008), la organización ya había operado más de treinta proyectos de alto impacto, alcance y cobertura, orientados al desarrollo emprendedor y empresarial y había transferido su modelo de incubación a quince incubadoras de empresas en el concierto nacional y diez en el internacional. Para esta misma época, la organización se había transformado en CREAME y contaba con una sede propia de 800 metros para prestar sus servicios y recibir a los nuevos emprendedores, ubicada en el edificio de la Red de Servicios Tecnológicos y Empresariales (TECNOPARQUE del SENA). En este lugar también se encuentran diez instituciones de ciencia, tecnología y emprendimiento de la ciudad de Medellín.

Sin embargo, el cambio estructural de la dinámica de la organización estuvo ubicado después de 2006, con la transformación de la incubadora en centro y la inclusión de nuevos servicios dirigidos especialmente a la operación de programas estratégicos de emprendimiento tradicional, de base tecnológica y rural, diseñados por instituciones, organizaciones $\mathrm{y}$ entes territoriales que buscan el desarrollo económico y productivo regional (CREAME, 2007).

Estos nuevos ejes de intervención amplían las características iniciales de la organización y la convierten explícitamente en Centro Integral de Servicios Empresariales (CREAME). Las características principales de la organización se pueden esbozar de manera concreta a partir de los interrogantes: ¿Qué es? ¿Qué hace? ¿Qué ofrece? ¿Con qué lo ofrece? ¿Cómo lo ofrece?¿A quién ofrece sus productos o servicios?

CREAME es hoy un Centro Integral de Servicios Empresariales de carácter mixto, pionero en Colombia en la generación de cultura emprendedora, la creación de nuevas empresas y el fortalecimiento de unidades económicas que salgan a los mercados nacionales $\mathrm{e}$ internacionales a competir activamente (CREAME, 2010).

Dentro de su quehacer, CREAME busca como estrategia alcanzar tres objetivos principales. Primero, la creación de empresas y el fortalecimiento empresarial a través de los centros de desarrollo empresarial y rural; segundo, mejorar las condiciones de financiación de los nuevos emprendimientos y los ya existentes por medio de Capitalia Colombia; tercero, 
operar de manera conjunta programas de emprendimiento con instituciones, organizaciones y entes territoriales de la región y el país.

Así, el Centro Integral de Servicios Empresariales desarrolla su labor y ofrecesus recursos y servicios en cinco líneas estratégicas, a través de un modelo en red por nodos que incluyen tres tipos de servicios y recursos (presencial y virtual) para apoyar a una masa crítica de emprendedores permitiéndoles elaborar su iniciativa de negocio hasta la creación y fortalecimiento de sus empresas, buscando resultados, impacto y rentabilidad, tal y como se detalla en la Tabla 7, la cual responde: con qué ofrece, cómo ofrece y a quiénes ofrece sus recursos y servicios.

Tabla 7. Descripción de la oferta de recursos y servicios de CREAME

\begin{tabular}{|c|c|c|}
\hline \begin{tabular}{|c|} 
¿Con qué ofrece \\
los servicios? \\
Tres tipos de \\
recursos y \\
servicios
\end{tabular} & \multicolumn{2}{|c|}{$\begin{array}{l}\text { 1) CREAME desarrollo empresarial: este centro de servicios fomenta el } \\
\text { emprendimiento en Colombia mediante tres servicios: la transferencia de } \\
\text { modelos de emprendimiento, el diseño y la operación de nodos empresaria- } \\
\text { les y el diseño, la gestión y la operación de programas de emprendimiento } \\
\text { a la medida de las organizaciones e instituciones. } \\
\text { 2) CREAME desarrollo rural: el centro gestiona de manera integral proyec- } \\
\text { tos agroindustriales y de emprendimiento social, por medio de servicios de } \\
\text { acompañamiento como socio empresarial, e incubación y formación espe- } \\
\text { cializadas. } \\
\text { 3) Capitalia Colombia (inversiones y finanzas): es un instrumento de co- } \\
\text { nexiones financieras inteligentes, que opera como banca de inversión para } \\
\text { la pequeña y mediana empresa prestando asesoría en finanzas corporativas } \\
\text { e instrumentos de intermediación de capital. }\end{array}$} \\
\hline $\begin{array}{c}\text { ¿Cómo ofrece } \\
\text { los recursos y } \\
\text { servicios? } \\
\text { Cinco líneas } \\
\text { de actividad } \\
\text { o ejes de } \\
\text { intervención }\end{array}$ & $\begin{array}{l}\text { 1. Creación de } \\
\text { empresas y forta- } \\
\text { lecimiento empre- } \\
\text { sarial }\end{array}$ & $\begin{array}{l}\text { CREAME dispone de dos pisos completos en el } \\
\text { Tecnoparque del SENA, dedicados a personas empren- } \\
\text { dedoras, equipado con oficinas, puntos de asesoramien- } \\
\text { to, aulas de formación, salas de reuniones y un espacio } \\
\text { de asesoramiento. } \\
\text { Los servicios que ofrece son: a) Convocatorias de ini- } \\
\text { ciativas empresariales y un acompañamiento integral de } \\
1 \text { año, denominado planeación o gestión, según el esta- } \\
\text { do en el que se encuentra, b) Asesoría en temas jurídi- } \\
\text { cos, gestión comercial, finanzas, modelación de nego- } \\
\text { cios, red de contactos y herramientas para el control y el } \\
\text { seguimiento integrado de la gestión empresarial, c) Alian- } \\
\text { zas con centros educativos, empresas privadas y cajas } \\
\text { de compensación familiar, con el fin de aplicar un modelo } \\
\text { flexible que promueva la creación de empresas alrededor } \\
\text { de un nicho de mercado específico, d) Incubación espe- } \\
\text { cializada, e) Formación especializada y f), Acompaña- } \\
\text { miento como socio empresarial. }\end{array}$ \\
\hline
\end{tabular}


Las políticas públicas de desarrollo empresarial e innovación desde la perspectiva G eovanny Perdomo Charry - J osé Enrique A rias Pérez

\begin{tabular}{|c|c|c|}
\hline & $\begin{array}{l}\text { 2. Asesorías en } \\
\text { finanzas corpo- } \\
\text { rativas y acceso } \\
\text { a capital. }\end{array}$ & $\begin{array}{l}\text { El centro dispone de una boutique financiera especiali- } \\
\text { zada para empresas con alto potencial de crecimiento, } \\
\text { así como el diseño de prácticas metodológicas y finan- } \\
\text { cieras que se adaptan a las necesidades de cada cliente. } \\
\text { Algunos servicios son: valoración de empresas y ges- } \\
\text { tión del valor, evaluación financiera de proyectos y em- } \\
\text { presas, diseño y desarrollo de herramientas de gestión y } \\
\text { planeación financiera, gestión y administración de fon- } \\
\text { dos de capital de riesgo, gestión y administración de } \\
\text { Redes de Inversionistas Ángeles, canalización de recur- } \\
\text { sos de financiación por medio de líneas de créditos es- } \\
\text { pecializadas y líneas de fomento, entre otros. }\end{array}$ \\
\hline & $\begin{array}{l}\text { 3.Operación de } \\
\text { programas y pro- } \\
\text { yectos con enti- } \\
\text { dades de desa- } \\
\text { rrollo territorial. } \\
\end{array}$ & $\begin{array}{l}\text { a) Diseño, gestión y operación de programas y proyec- } \\
\text { tos de emprendimiento a la medida de las organizaciones } \\
\text { e instituciones locales, regionales y nacionales. } \\
\text { b) Nodos o centros de atención que se crean en alianzas } \\
\text { con centros educativos, empresas privadas y cajas de }\end{array}$ \\
\hline & $\begin{array}{l}\text { 4.Posicionamien- } \\
\text { to de la marca } \\
\text { CREAME. }\end{array}$ & $\begin{array}{l}\text { compensación familiar, con el fin de aplicar un modelo } \\
\text { flexible que promueva la creación de empresas y } \\
\text { posicione la marca CREAME. } \\
\text { c) Transferencia de modelos de emprendimiento } \\
\text { estandarizados para la ejecución de programas de } \\
\text { emprendimiento social, agroindustrial, de valor agrega- } \\
\text { do o de base tecnológica. }\end{array}$ \\
\hline & $\begin{array}{l}\text { 5.Aumento de la } \\
\text { rentabilidad a tra- } \\
\text { vés de las empre- } \\
\text { sas propias. }\end{array}$ & $\begin{array}{l}\text { El modelo de operaciones de CREAME está soportado } \\
\text { en dos unidades de negocio: de un lado, el desarrollo } \\
\text { productivo y empresarial que tiene dos líneas de trabajo } \\
\text { correspondientes a consultoría para el desarrollo de te- } \\
\text { rritorios y emprendimiento rural y social, y del otro, nue- } \\
\text { vos negocios, que tienen operación con Capitalia Co- } \\
\text { lombia y la Certificadora. }\end{array}$ \\
\hline $\begin{array}{c}\text { ¿A quién ofrece } \\
\text { los recursos y } \\
\text { servicios? }\end{array}$ & \multicolumn{2}{|c|}{$\begin{array}{l}\text { El modelo de operaciones de CREAME está soportado en dos unidades de } \\
\text { negocio: de un lado, el desarrollo productivo y empresarial que tiene dos } \\
\text { líneas de trabajo correspondientes a consultoría para el desarrollo de terri- } \\
\text { torios y emprendimiento rural y social, y del otro, nuevos negocios, que } \\
\text { tienen operación con Capitalia Colombia y la Certificadora. }\end{array}$} \\
\hline
\end{tabular}

Fuente: elaborado por los autores, con base en CREAME (2011).

La tabla anterior muestra una orientación del centro hacia la generación de nuevos ingresos por medio de la operación de programas y proyectos territoriales e institucionales y la creación de nuevos negocios, desarrollando labores más transversales de conformación de empresas y no de incubación propiamente dicha en la ciudad y la región, que son actualizados de forma permanente teniendo en cuenta las demandas del entorno. 
Descripción y cronología de las políticas y programas de emprendimiento

En el contexto anterior se puede señalar que el Estado tradicionalmente establece un marco jurídico del sistema de desarrollo urbano, social y económico. Controla un gasto importante en infraestructura para la malla vial y la red de transporte nacional, regional y local.

El Estado también asigna la mayor parte de los fondos públicos para inversión social y seguridad del país y la ciudad. Adicionalmente hay una serie de políticas, planes, programas y fuentes de financiación que se dirigen específicamente al desarrollo económico y empresarial del país y la región, muchos de los cuales son importantes para Medellín (BID, 2011).

De ahí que las "políticas públicas para esta investigación se asuman como configuraciones de los actores" (Muller, 2006, p. 67), debido a que su construcción ha ido evolucionando en el tiempo. Es decir, en las dos primeras décadas (1980 y 1990) su elaboración era de arriba a abajo (institucionalmente) y durante 2000 se ha iniciado un proceso de construcción en doble vía, de abajo a arriba y viceversa; una prueba de ello, es el SNCTI, el cual está compuesto por organizaciones públicas, privadas y mixtas que interactúan flojamente entre sí, pero están en proceso de consolidación de sus relaciones.

Por consiguiente, las políticas públicas no son sólo un proceso de decisión, sino un lugar donde determinada comunidad construye su relación con el entorno, configurando la realidad que quiere intervenir (Muller, 2006). Dicha interacción entorno-organización, se ha tejido intermitentemente entre CREAME y la Alcaldía de Medellín, como lo expresa el empleado número 9:

Pues eso tendría que dividirlo como en varios ámbitos, lo que es lo (sic) nacional, lo departamental y ya pues lo local; por otro lado yo digo que aquí ha habido mucho desorden en las políticas a nivel nacional porque aquí estamos inventando la rueda en cada región... Entonces, yo hablaba en estos días con una persona, que aquí falta más articulación en todo el tema de emprendimiento y posiblemente por eso digo que falta más relacionamiento corporativo con otro tipo de actores y yo sé que por ejemplo, la incubadora ha venido haciendo ese trabajo y lo ha hecho muy bien, por ejemplo con la Alcaldía de Medellín con todo este tema de ciudad cluster.

La percepción anterior muestra que aunque existe diversidad de políticas y programas formulados y legitimados en el campo del emprendimiento y la innovación dentro de los planes de desarrollo nacional, regional y local (Meny \& Thoening, 1992), aún falta un verdadero relacionamiento de los actores y el SRI en Medellín-Antioquia, para lograr ser una ciudad y región innovadora.

De hecho, el diseño de políticas y programas de emprendimiento e 
innovación se da de forma explícita en el año 2000, con ciertas características particulares que permiten suponer que su elaboración estuvo dirigida autónoma y gradualmente por la Gobernación de Antioquia y la ciudad, con el apoyo indirecto de CREAME, como se puede apreciar en la concepción que tiene el emprendedor número 1 sobre el diseño de políticas y programas en este campo.

Antes CREAME ha sido el creador de todos estos programas, CREAME fue pionero en temas de emprendimiento, es un referente. CREAME mandó al doctor Darío Montoya al SENA, creó el fondo Emprender, creó una masa crítica de emprendimiento importante en el país; después sale el equipo CREAME a montar el Parque del Emprendimiento. CREAME incubó un sistema de emprendimiento muy importante en el país; además de las incubadoras que creó en otras partes.

Lo cual evidencia que entidades como CREAME, participan y lideran propuestas públicas de desarrollo empresarial, que luego se convierten en programas de desarrollo económico de la ciudad.Ello refleja la transición hacia modelos de gestión pública del tipo modus vivendi $o$ negociado de acción o de gobernanza.

Por tanto, las políticas y programas de desarrollo empresarial en Colombia, Antioquia y Medellín, han presentado tres cambios fundamentales de evolución del sistema socioeconómico, científico y tecnológico. El primero es visto como el inicio del cambio institucional: se desarrolló durante los años ochenta y su foco de acción estuvo orientado a mejorar los índices de pobreza e inequidad social, así como a reactivar la economía colombiana afectada por una fuerte crisis producto de la violencia y los bajos niveles de desarrollo del aparato productivo nacional.

El segundo se concibe como la aceleración del cambio institucional: surgió en la década de 1990 y se ocupó principalmente por alcanzar la paz y fortalecer la inclusión social de la sociedad civil, así como el inicio y la ejecución tímida de políticas y planes de desarrollo económico vía competitividad, con el fin de mejorar el aparato productivo nacional para exportar o atraer inversores internacionales. Este periodo de desarrollo estuvo destinado a fortalecer la autonomía de las regiones enmarcadas en la nueva Constitución Política de 1991.

Finalmente, la protección y estabilización de la institucionalidad, que busca el desarrollo económico y empresarial, ligado al bienestar social de la población, cuyo énfasis mayor está en fortalecer el desarrollo empresarial y la competitividad de las empresas a través de la inversión en CT\&I, se promueve con el fomento de la relación e interacción entre los distintos actores de la sociedad civil, como se observa en la tabla de relación sintética de las políticas y programas de desarrollo empresarial e innovación (Tabla 8), siguiendo las construcciones analíticas (génesis, adolescencia, adultez y madurez) expuestas por Hoffman (2001) y Eshun (2004). 


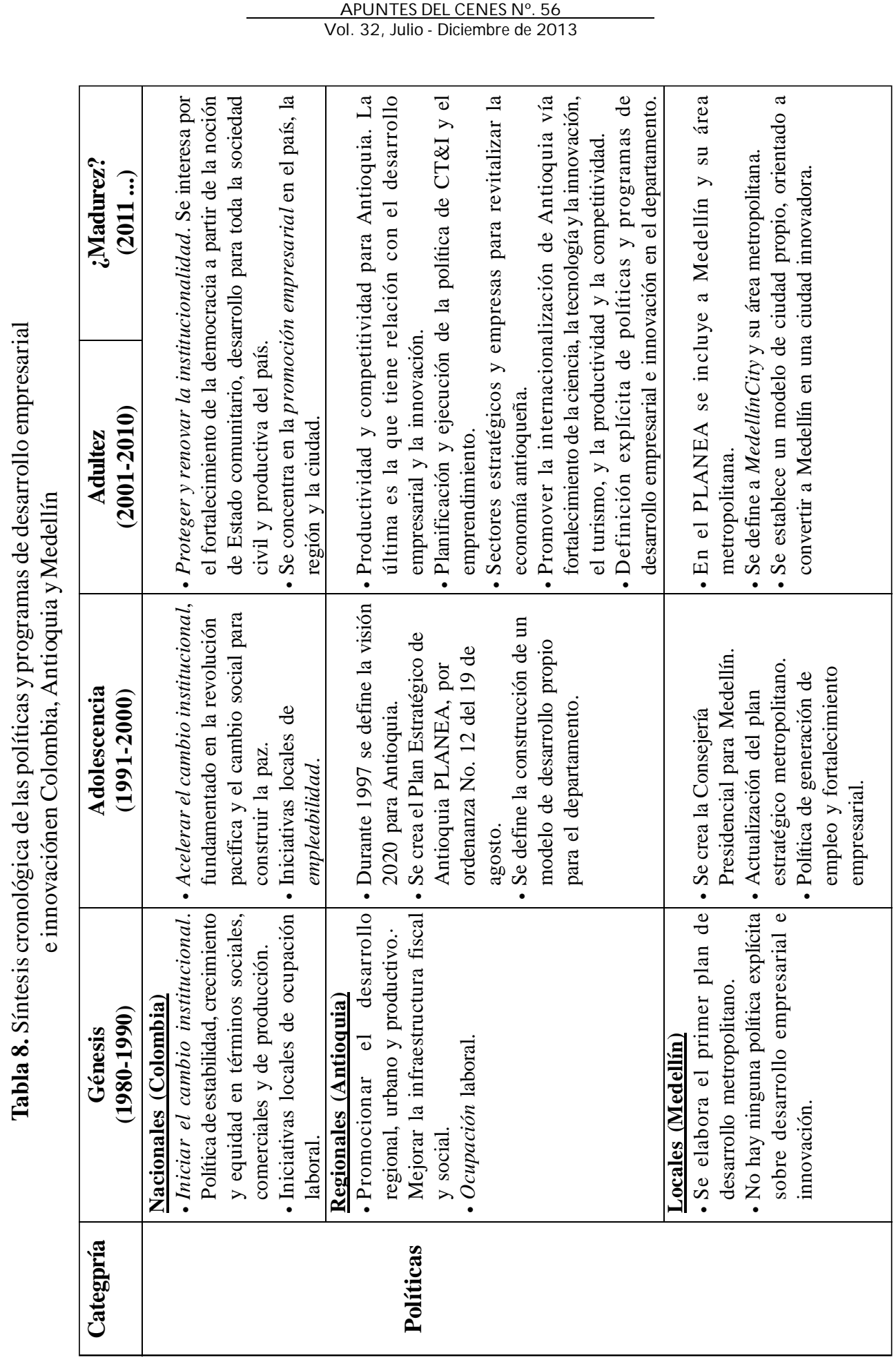


Las políticas públicas de desarrollo empresarial e innovación desde la perspectiva

Geovanny Perdomo Charry - J osé Enrique A rias Pérez

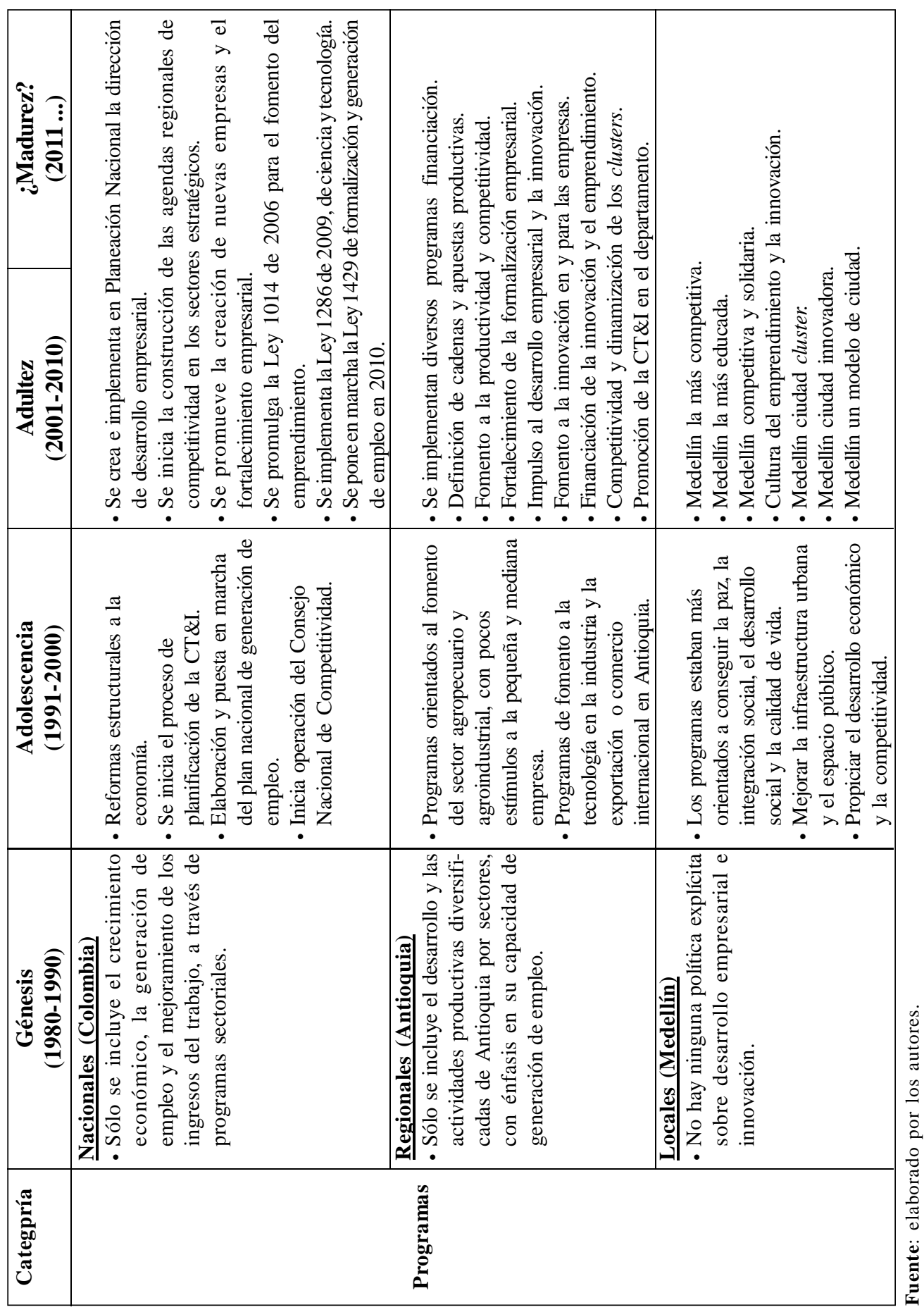

198 
En la tabla anterior se hace visible que las políticas y programas se han transformado y evolucionado desde su concepción, coordinación y ejecución de actividades de emprendimiento e innovación, como algo construido conjuntamente entre el sector público y privado en Medellín y Antioquia, mediante la participación de diferentes actores del sistema regional de innovación (SRI) en su diseño e implementación.

Por tanto, la manera de diseñar, formular, operar y evaluar las políticas públicas y los programas de acción ha ido cambiando paulatinamente de un gobierno centralizado a uno descentralizado, en "donde la mayor parte de la producción de políticas se ha desplazado a los entes no gubernamentales" (Peters, 2004, p. 69); es decir, con una vinculación directa de actores privados en el diseño de las políticas, pero orientados, dirigidos y evaluados por el Estado.

El proceso de dirección de una comunidad determinada "no es equivalente a una sola acción directiva del gobierno sino del conjunto social" (Ramírez, Vargas \& De la Rosa, 2011, p. 39), que se deriva de la construcción de capacidades institucionales en materia de desarrollo económico, empresarial y de innovación en la ciudad de Medellín y el departamento de Antioquia.

Incluso se observa que la política económica nacional está centrada en el bienestar social y la equidad para la población colombiana, con poco interés en el desarrollo empresarial y la ciencia, la tecnología y la innovación (CT\&I) a nivel departamental y municipal. Sin embargo, en los últimos diez años ha habido un interés particular por aplicar la Constitución Política colombiana en su componente de autonomía regional, con el fin de que cada región reorganice sus estrategias e iniciativas de planificación y ejecución de políticas y programas a escala departamental, especialmente en relación con el emprendimiento y la innovación, así como con la productividad y competitividad empresarial en el contexto nacional e internacional.

Por su parte, en el ámbito internacional se busca incrementar las actividades regionales y competir de manera global, por medio de diversidad de acciones estratégicas coordinadas por el departamento de Antioquia y la Alcaldía de Medellín durante los últimos veinte años. Este proceso ha estado orientado fundamentalmente a mejorar la calidad de vida y el bienestar social de la comunidad mediante el fomento al emprendimiento, la investigación, el desarrollo tecnológico, la innovación y el fortalecimiento empresarial.

Precisamente, durante la década de 2000 se expone de forma explícita el diseño y la operación de políticas y programas de ciencia, tecnología e innovación (CT\&I) y desarrollo empresarial en Colombia, no sólo desde la formulación, sino desde la implementación creciente de medidas de fomento del espíritu emprendedor y la innovación. 
Por eso, si se reconoce que una organización y un programa son ante todo espacios para negociar más o menos institucionalizados, entonces deben concurrir muchas mediaciones para que se alcance un compromiso (Monnier, 1992). Dicho comportamiento ha hecho que Medellín y CREAME replanteen y redefinan gradualmente las políticas y programas por "fases u etapas (formulación, ejecución y evaluación), en respuesta a la demanda social o de mercado, en términos de gobernanza" (Peters, 2004, p. 68).

La gobernanza se percibe como un "conjunto de mecanismos que proveen servicios públicos a ciertos espacios sociales" (Peters, 2004, p. 69), que se conciben, en otros términos, ya no desde un punto de vista secuencial, sino en virtud de la naturaleza de las relaciones entre los actores sociales de Antioquia y Medellín. En consecuencia, el departamento de Antioquia y la ciudad de Medellín han logrado desarrollar cuatro elementos clave 3 para fomentar el espíritu emprendedor en un contexto específico, mediante la superación gradual y superficial de tres dilemas básicos en los procesos de planificación (Castells \& Vilaseca, 2007).

1. El dilema entre la adopción de medidas horizontales de fomento a la creación de empresas y medidas de apoyo selectivas, dirigidas a grupos específicos susceptibles de recibir una atención preferente.

2. El dilema entre apoyar la creación de empresas, de cualquier índole, que estimulen la auto-ocupación, o proporcionar ayuda preferente a aquellas firmas que, por el tipo de actividad que desarrollan o su contenido tecnológico, presentan a priori un mayor potencial de crecimiento, consolidación y arrastre (empresas gacelas).

3. El dilema entre emplear la eficiencia económica y la social como criterio preferente en la distribución de los recursos públicos.

Tales disyuntivas han sido desarrolladas por algunas entidades territoriales de Medellín que promueven el emprendimiento por medio del sistema regional de innovación (SRI), con la inclusión de instrumentos como los parques tecnológicos, las unidades de emprendimiento y las incubadoras de empresas. Entre otras cosas, este último instrumento ha perdido reconocimiento nacional, debido a que las incubadoras no cuentan con el apoyo técnico y económico suficiente para desarrollar su objeto social, por tanto, esto hizo que la IEBTA se transformara en CREAME durante el 2006 y se dedicara a la consultoría empresarial.

i) Eliminación de los obstáculos a la creación de empresas, ii) mejor equilibrio entre los riesgos y las recompensas a la iniciativa empresarial, iii) fomento de la capacidad y las competencias empresariales, iv), mayor accesibilidad de la iniciativa empresarial al conjunto de la sociedad. 
Por supuesto, la relación de actores y sistema confluye en el diseño y operación de políticas y programas de desarrollo empresarial e innovación, que han venido legitimando de manera progresiva la cultura emprendedora en la ciudad, en términos de Monnier (1992). En otras palabras, el éxito que ha tenido la política de emprendimiento e innovaciónes producto de la acción decidida de los gobernantes y los actores del sistema regional de innovación (SRI) de Medellín y Antioquia.

La actualización permanente de las actividades y los servicios ofrecidos por los actores que pertenecen al sistema, es producto de una inquietud institucional (políticas y programas) clara para lograr los propósitos de desarrollo económico y empresarial de forma eficiente (Castells \& Vilaseca, 2007).

De allí que la capacidad institucional sea vista como un complejo entramado de recursos, mecanismos y dispositivos de orden técnico y organizacional, pero de naturaleza política, que un gobierno como el de Medellín dispone para la conducción de la acción social dirigida al desarrollo y bienestar de la sociedad civil, histórica y socialmente determinada (Ramírez, Vargas \& De la Rosa, 2011).

\section{CONCLUSIONES}

Desde el año 2001, las políticas públicas de desarrollo empresarial e innovación se encuentran en un estado de adultez, surgidas predominantemente en el contexto regional, por la convergencia de diversos actores, especialmente privados, que juegan un papel importante en el diseño y ejecución, si bien el seguimiento y la evaluación siguen principalmente en manos del Estado; lo cual coincide con los rasgos del modelo de gestión pública modus vivendi o negociado de acción, y de la gobernanza.

Sin embargo, si bien en el ámbito regional son evidentes los avances en cuanto a la transición hacia modelos de gobernanza en materia de políticas públicas de desarrollo empresarial e innovación, son también notorias las dificultades para "negociar la acción" con los actores nacionales que tienen injerencia en los procesos de formulación, lo cual, en ocasiones, genera redundancias y tensiones entre lo regional y lo nacional.

En ese orden de ideas, CREAME es una de las instituciones del sistema regional de CT\&I, resultante de la articulación público-privada, que, al mismo tiempo, se reconoce como un referente en el diseño de nuevas políticas públicas de desarrollo empresarial e innovación, y en un actor de notoria incidencia en sus etapas de formulación, ejecución y evaluación.

De otra parte, la creciente participación de actores no gubernamentales en la formulación de las políticas, evidencia la legitimación del accionar de los mismos, cuya vinculación constituye un mecanismo para subsanar la racionalidad limitada y un medio para conectar las políticas de desarrollo empresarial e 
innovación con las demandas sociales o del mercado.

La evolución de la política pública de desarrollo empresarial e innovación, ha transformado a CREAME, dedicada en un inicio a la incubación de empresas, en una institución que también ofrece servicios de consultoría, especialmente porque este instrumento ha perdido reconocimiento nacional,debido a que no logra brindar el apoyo técnico y económico suficiente para desarrollar eseprimer objeto social.
Futuras líneas de investigación, pueden orientarse al estudio de las políticas públicas, entendidas como configuración de actores que negocian la acción en el marco de los propósitos nacionales y regionales, en ocasiones antagónicos; en consecuencia, sería pertinente identificar los inhibidores o impulsores de los acuerdos y las tensiones, así como poder estudiar el desarrollo de otros actores del sistema producto de las políticas públicas en materia de desarrollo empresarial e innovación.

\section{R eferencias}

Abduh, M. D., Souza, C., Quazi, A. \& Burley, H. T. (2007). Investigating and classifying clients' satisfaction with business incubator services. Managing Service Quality, 17 (1), 74-91.

Allen, D. N. \& Rahman, S. (1985). Small business incubators: a positive environment for entrepreneurship. Journal of Small Business Management, $23(3), 12-22$.

Allen, D. N. \& McCluskey, R. (1990). Structure, policy, services, and performance in the business incubator industry.Entrepreneurship: Theory and Practice, 15 (2), 61-78.

Arellano, D. \& Cabrero, E. (2005). La Nueva Gestión Pública y su teoría de la organización: ¿son argumentos antiliberales? Justicia y equidad en el debate organizacional público. Gestión y política pública, 14 (3), 599-619.
BID (2011). Medellín: transformación de una ciudad. Alcaldía de Medellín 2008-2011. Medellín, Colombia: Banco Interamericano de Desarrollo.

Campbell, C., Berger, D., Janus, J. \& Olson, K. (1988). Change agents in the new economic: business incubators and economic development. Minneapolis, $\mathrm{MN}$ : Hubert Humphrey of Institute Public Affairs.

Castells, O. M. \& Vilaseca, R. J. (Edit.) (2007). Entorno innovador, iniciativa emprendedora y desarrollo local. España: Octaedro,

CREAME (1998). Informe anual de gestión, presentado a la asamblea general de la Corporación Incubadora de Empresas de Base Tecnológica en el mes de marzo. Medellín, Colombia: CREAME. 
CREAME (1999). Informe anual de gestión, presentado a la asamblea general de la Corporación Incubadora de Empresas de Base Tecnológica en el mes de marzo.Medellín, Colombia: CREAME.

CREAME (2006). Informe anual de gestión, presentado a la asamblea general de la Corporación Incubadora de Empresas de Base Tecnológica en el mes de marzo. Medellín, Colombia: CREAME.

CREAME (2007). Informe anual de gestión, presentado a la asamblea general de la Corporación Incubadora de Empresas de Base Tecnológica en el mes de marzo. Medellín, Colombia: CREAME.

CREAME (2011). Portafolio de Servicios del Centro Integral de Servicios Empresariales. Medellín, Colombia: CREAME.

Crozier, M. \& Friedberg. (1990). El actor y el sistema. Las contradicciones de la acción colectiva. México: Patria. Traducción de la obra en Francés L'acteur et le systeme. Les contraintes de l'acttion collective. Editada en 1977 por Éditions du Seuil.

Eshun Jr., J. P. (2004). ¿Where do business incubator come from? the origins, evolution and institutionalization of business incubator: evaluating performance and assessing outcome. $\mathrm{PhD}$ thesis submitted to the University of Columbia, New York.

Eshun Jr., J. P. (2009). Business incubator as strategy.Business Strategy Series, 10 (3), 156-166.

Garrido, M. (2003). Sistema Nacional de Innovación y Centros de Desarrollo Tecnológico en Colombia. Primer
Congreso Iberoamericano de Centros Tecnológicos. Valencia, España.

Hackett, S. M. \& Dilts, D. M. (2004a). A systematic review of business incubation research. The Journal of Technology Transfer, 29 (1), 55-82.

Hackett, S. M. y Dilts, D. M. (2004b). A real options-driven theory of business incubation. The Journal of Technology Transfer, 29 (1): 41-54.

Hansen, M.T., Cresbrough, H.W., Nohria, N.\&Sull, D.N. (2000). Networked incubators: hosthouses of the new economy. Harvard Business Review, 78 (5), 74-84.

Hoffman, A. J. (2001). A road map of corporate environmentalism. In From heresy to dogma, an institutional history of corporate environmentalism. Stanford, CA: Stanford University Press.

Malaver, R. F. \& Vargas, P. M. (2005). Políticas y avances en la ciencia, la tecnología y la innovación en Colombia 1990-2005. Cuadernos de Administración, 18 (30), 39-78.

Malaver, R. F. \& Vargas, P. M. (2006). La innovación en Colombia 1990-2004. Innovación y Ciencia, 13 (3), 40-47.

Meny, Y. \& Thoening, J. C. (1992).Las políticas públicas. F. Morata (trad.). Barcelona: Ariel.

Monnier, E. (1992). Evaluations de l'action des pouvoirs publics. Paris: Económica.

Monroy, S. E. (2006). Nuevas políticas y estrategias de articulación del sistema de ciencia y tecnología e innovación colombiano. Revista Innovar, 16 (28), 157-172. 
Montenegro, T. I. (2007). Existe un Sistema Nacional de Innovación en Colombia. Bogotá: Colciencias.

Muller, P. (2006). Las políticas públicas. Bogotá: Universidad Externado de Colombia.

Robledo, J. \& Echavarría, E. S. (2006). Un Sistema Nacional de Ciencia, Tecnología e Innovación: hacia la necesaria integración de la investigación y la innovación. Innovación y Ciencia, 13 (3), 34-39.

Orozco, P. (2006). Un aporte a la discusión sobre los Sistemas Nacionales de Ciencia, Tecnología e Innovación. Innovación y Ciencia, 13 (3), 34-39.

Peters, G. B. (2004). De la administración pública a la gobernanza. En: M. Pardo (comp.). Cambios en la naturaleza de la administración pública: de las preguntas sencillas a las respuestas difíciles. México: El Colegio de México, Centro de Estudios Internacionales.

Ramírez, M. G., Vargas, L. G. \& De la Rosa, A. A. (2011). Estudios organizacionales y administración. Contrastes y complementariedades: caminando hacia el eslabón perdido. Revista Fórum Doctoral, 3, 7-51.
Rosas, C. J. (2011). Gestión y evaluación en la institución municipal. Coordinación, concurrencia, interrelaciones $y$ políticas públicas para el desarrollo local. Concurso de oposición. Convocatoria: CO.A.CSH.a.005.10, México.

Sherman, H. \& Chappell, D.S. (1998). Methodological challenges in evaluating business incubator outcomes. Econ.Dev, 12 (4), $313-321$.

Smilor, R. \& Gill, M.D. Jr. (1986). The new business incubator: linking talent, technology and know-how. Lexington, MA.: Lexington Books.

Tsai, F. S., Hsieh, L. H. Y. Fang, S. C. \& Lin, J. L. (2009). The co-evolution of business incubation and national innovation systems in Taiwan. Technological Forecasting and Social Change, 76 (5), 629-643.

Verma, S. (2004). Success factors for business incubators: an empirical study of canadian business incubators. MBA thesis submitted to the Eric Sprott School of Business Carleton University, Ottawa, Ontario.

Yin, R. K. (2003). Case study research. Design and methods. (3rd. Ed.). Thousand Oaks: s.n. 